\title{
Dorsal Hand Vein Analysis For Security Systems
}

\author{
Humairah Hamid $^{1}$, V. K. Narang ${ }^{2}$, Dr. Priti Singh ${ }^{3}$ \\ ${ }^{1}$ M.Tech student, Department of Electronics and Communication Engineering, Amity University Haryana \\ ${ }^{3}$ Professor (Dr), Head Of the Department ECE - EEE, Amity University Haryana \\ ${ }^{1}$ Humairahhamid9@gmail.com \\ 3psingh@ggn.amity.edu \\ ${ }^{2}$ Professor, Director Amity School of Engineering and technology, Amity University Haryana \\ 2vknarang@ggn.amity.edu
}

\begin{abstract}
Biometric systems such as the fingerprint based, iris based or face recognition based are being widely used for personal verification and identification. The systems based on the vein pattern analysis is the most recent approach falling in this category. It uses the large network of blood vessels underneath the skin as the feature for identification. This system provides contactless image acquisition and the vein pattern details can't be forged easily, thus making is better as compared to the other techniques. In the proposed system, the translational effects are minimized by taking the region of interest (ROI) at center of hand. This is done by detection of centroid. The key features used for matching purpose are the line segments extracted using Hough Transform. Finally, the authentication of the users is proved on the basis of the matching algorithm. Here the Modified Hausdorff Distance is used for the matching purpose which gives the False Acceptance Rate (FAR) of $0.2 \%$ and False Rejection Rate (FRR) of $0.1 \%$. Analysis is performed on the images acquired from CIR biometrics database.
\end{abstract}

Keywords: Image processing, Identification and verification of dorsal hand vein, Infrared imaging (IR), Hausdorff distance.

\section{INTRODUCTION}

Biometrics is the science and technology of measurement and analysis which works on the principle of measurable physiological or behavioral characteristics. It is being considered as the framework of extremely secure identification and private verification solutions. The increase of the security breaches and frauds have led to the development of more secure biometric recognition system. As a result, the need for extremely secure identification and verification technologies is increasing. Biometric based recognition system has several applications like secure electronic banking, investing, money transactions, enforcement and health and social services. Biometric analysis is divided into two phases: Enrollment phase and recognition phase. Enrollment includes initial profile measurements for information storage. It is usually slow and considers multiple measurements. In recognition, the matching of the selected profile with the stored template is done. Examples of biometric system are face, fingerprint, iris, retinal scan etc. These systems have their own benefits and drawbacks. One of the major drawbacks of face recognition based systems is that they are not user friendly. The user needs to make contact with the system to acquire the image. In iris based systems, eye is exposed to light radiation causing inconvenience to the user. However recently, vein pattern recognition has become a brandnew technique for identity.

The vein based system have various advantages as compared to the other systems. They offer contactless image acquisition. The subcutaneous vascular pattern called hand vein that is on the rear of the hand is extremely troublesome to forge. Therefore, it ensures promising biometric. The hand veins on the dorsum are basically of two types: cephalic and basilica veins. The basilica veins are the vein cluster connected to the surface of the hand. It consists of the higher limb of the rear of the hand. The cephalic veins are the cluster of veins connected with the elbow of the hand [6]. The shape of the hand veins slightly changes with the change in the length of the body from childhood. Therefore, no major changes occur within the adult life that makes the vein pattern quite stable for the people of age group 20 - 50. After that, with the decrease in the bones strength and muscle, the system begins to shrink. These changes minutely affect the vein patterns making them loose and thus affecting their size. Some diseases that influence the vascular system are sugar levels, high blood pressure, disorder and tumors. They modify the veins making them thick or skinny. Considering the above factors, we have optimized our technique for better results.

Vein pattern biometrics is a huge area of research. Various researchers have contributed to this field. In 1991, the first biometric system proposed by P.Macgregor and R.Welford used the filments made of tungsten for illumination. The vein structure was plotted manually to extract features but this was not helpful for practical purpose [1]. In 2008, Li Xueyan and Guo Shuxu used the thermal imaging for image acquisition. Thermal imaging is useful under certain temperature condition only, therefore this technique got limited to some systems 
only [4]. In 2009, Ajay Kumar, K. Venkata Prathyusha proposed the use of knuckle tips as the key feature for identification. It had a disadvantage that displacement of the hand may sometime not cover the kuckle tips [5]. Ricardo Janes, Augusto Ferreira Brand in 2014 used the curvelet transform for feature detection which introduced some losses in the image [6]. Later in 2015, M. Rajalakshmi, R. Rengaraj , used the delaunay's principle for finding vein ending and bifurcation points as the key features. This principle is difficult to implement if there are less number of points. This fails in case the extracted veins do not have huge number of points extracted [7]. On the other hand, the proposed system uses the line segments for authentication which give more accurate results as compared to previous work [8].

\section{PROPOSED SYSTEM}

This paper presents an approach to authenticate the individuals based on their dorsal hand vein pattern. This approach utilizes the dorsal hand vein images which are acquired form a low cost, contactless Near Infrared (NIR) camera.

The architecture of the system is presented below: (fig 1)

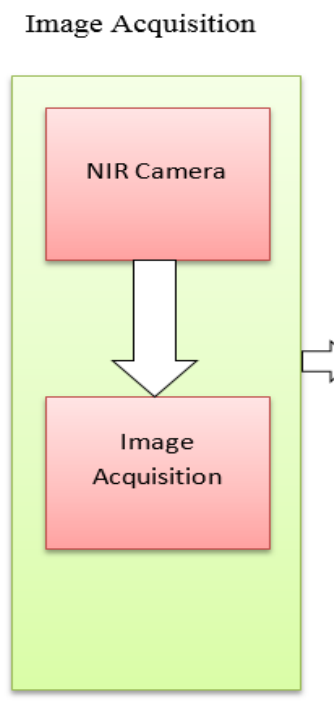

Pre-Processing

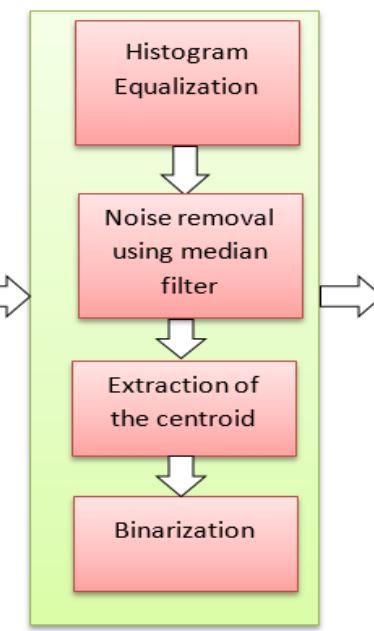

Fig 1. Block diagram of the system
Feature extraction and matching

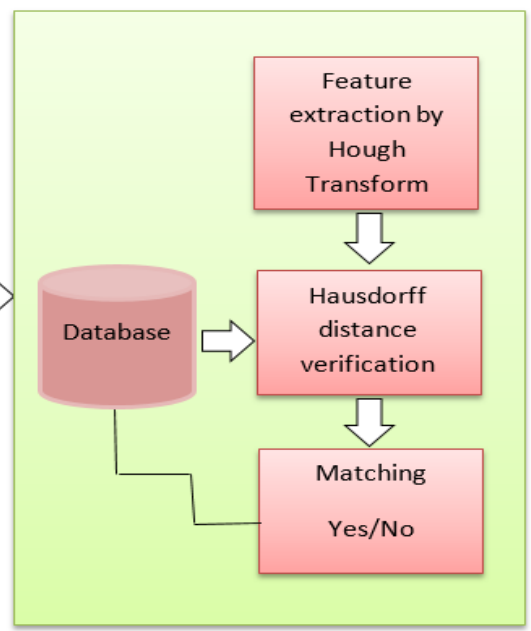

\section{A. Image acquisition}

Image acquisition can be done in two ways. Either by Far Infrared (FIR) imaging or Near Infrared (NIR) Imaging. FIR light having wavelength in the range of $400 \mathrm{~nm}$ to $700 \mathrm{~nm}$ works on the principle that the veins have higher temperature than the surroundings and a thermal imaging camera can be used to get the image. But this systems had a disadvantage that when used in the open region, it can introduce noise in the image and thus requires more preprocessing. The NIR image acquisition technique has number of advantages over the other techniques. NIR light having wavelength of 700nm - 900nm can transmit through the skin upto 3mm depth. The veins have deoxygenated blood in them and the NIR light is completely absorbed by the blood. Due to this, the veins appear to be darker than the rest of the region in the image taken form NIR camera. This makes the veins clearly visible as dark foreground and skin as light background. Here the NIR imaging is used. Since such a process is costly and general webcam doesn't give the good quality images suitable for analysis. The images have been taken from a free web source CIR biometrics database. It consists of 50 images of different users. Fig 2 shows one of the database images.

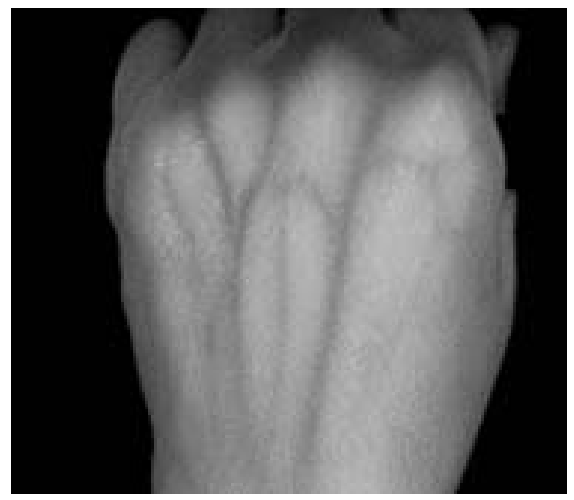

Fig 2. Acquired Images 


\section{B. Preprocessing and extraction of Region Of Interest (ROI)}

The captured image is converted to a grayscale image. This is important as the gray images can be manipulated easily at the later stages. Further the histogram equalization is performed in order to improve the contrast of the image. This helps in getting the details accurately and more clearly. The images acquired from the NIR camera may not necessarily have zero noise level. In order to extract a clear vein pattern, noise removal is required. A median filter is employed for this purpose. Then thresholding is done in order to get a clear view of background and foreground. This is done by Otsu method. It is a global thresholding method which computes a threshold value that is used to transform a grey image to the binary image. Fig 4 shows the result of thresholding.

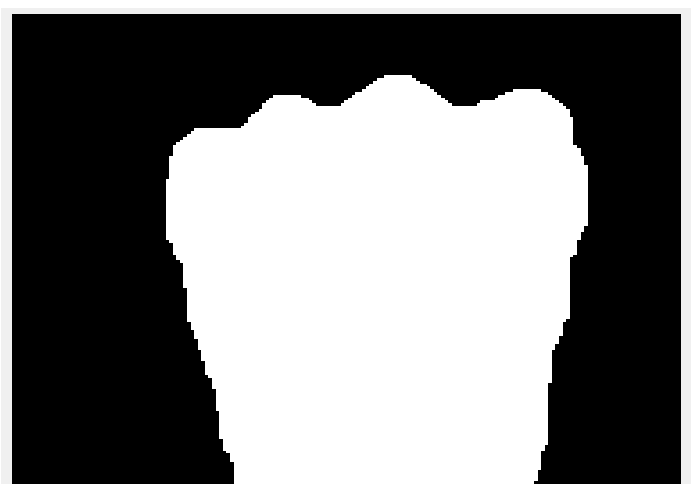

Fig 3. Thresholding

After thresholding, the main aim is to select the Region of Interest which is done by extracting the centroid. A uniform area of $200 * 200$ is grown around the centroid and is used for further analysis. This is the optimized region of interest as it includes the necessary area for analysis. The features to be analyzed are extracted from this region only. Under normalized conditions, ROI should include the same region when different dorsal hand vein images are considered. The below figure (fig 4) shown the region of interest with centroid detection.
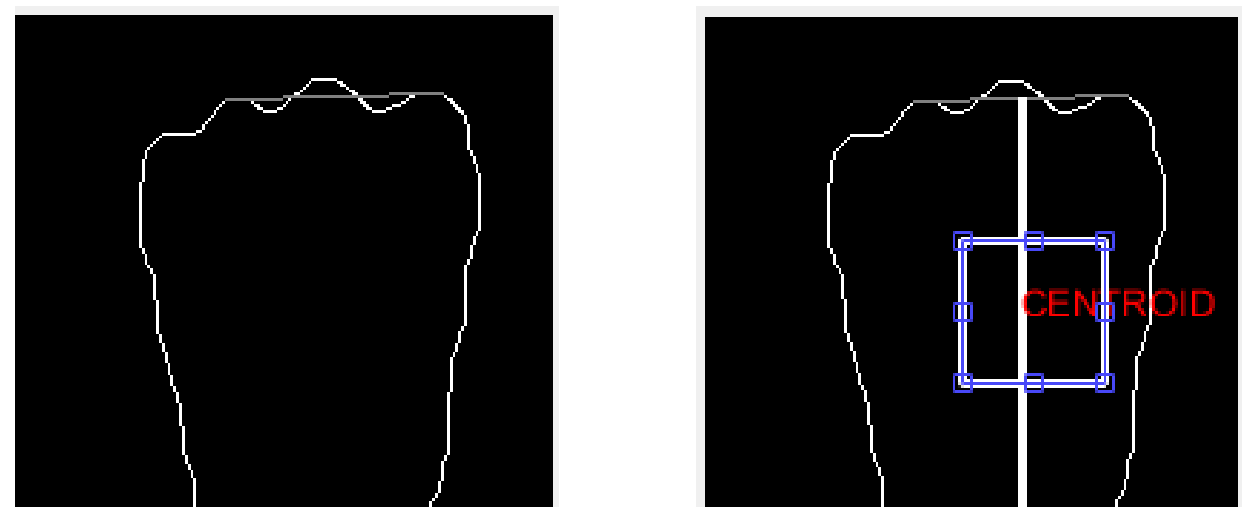

Fig 4. Region Of Interest selection

\section{Feature extraction}

For feature extraction Line Edge Map (LEM) technique is used. It works by first binarizing the image, then skeletonization and finally the line segment generation.

Once the preprocessing is done, the quality of the image improves but the vein pattern is still surrounded by many faint white regions. In order to obtain a better vein pattern, separation of the vein pattern from the background of the image is necessary. For this, the global thresholding by 'Otsu' method has been used. It works by creating a binary image by replacing all the values above a threshold with $1 \mathrm{~s}$ and setting all other values to 0s. This creates a binarized image. Fig 5 shows the binarized veins of selected region. 


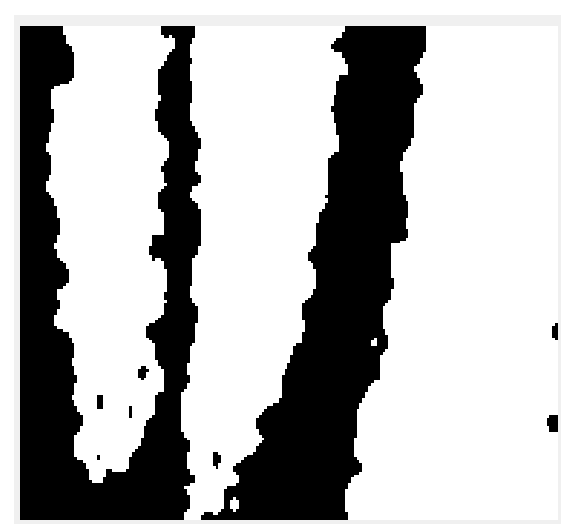

Fig 5. Binarization

After binarization, the vein may appear thick and it may be difficult to extract the required features. In order to obtain the clear features for matching purpose, skeletonization has been applied. This extracts the skeleton of the vein patterns successfully and preserves the shape of the vein patterns. This produces a single pixel wide skeleton which is easy to analyze as shown in fig 6.

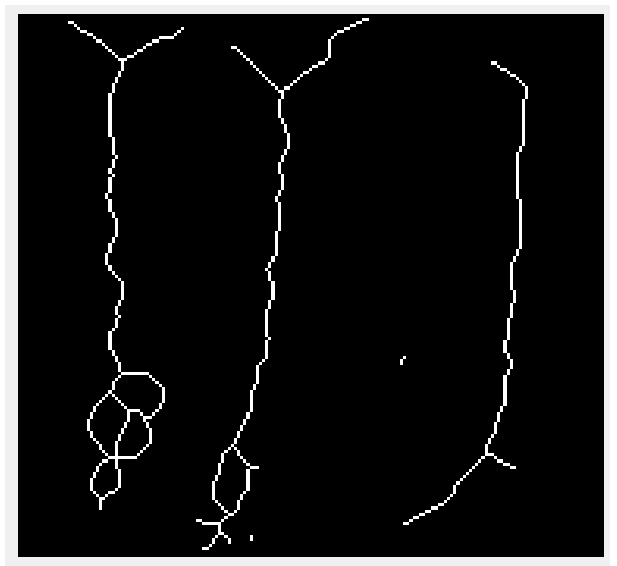

Fig 6. Skeletonization

Skeletonization gives the perfect size for the vein analysis. The vein structure thus formed can be represented by the number of line segments. These line segments are used as a feature to match the vein patterns and hence can be used to identify a person. To obtain the line segments from the vein pattern, the Hough transform has been used. Hough transform works by first detecting the Hough peaks in the vein pattern and then draws it in the form of a structural array. The line segments are generated depending upon the length specified. Here minimum length for the line segments is 20 (arbitrary units). All the line segments below this threshold are ignored. Fig 8 shows the line segments generated

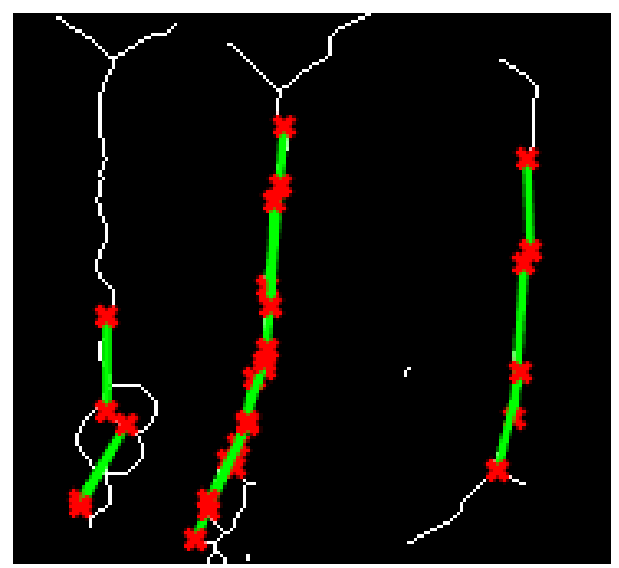

Fig 7. Line segment generation 


\section{Matching algorithm}

In order to verify the performance of the system, the generated line segments are matched using the Modified Hausdorff Distance algorithm (MHD). It is a measure for comparing the similarity of two point sets. MHD works by computing the forward and backward distance between the two point sets and gives the output as the minimum of both. Here the threshold for MHD is set to 10. If the distance between the two pointsets is less or equal to 10 , then it indicates that the user is the authorized one. This is implemented using a GUI as shown below:

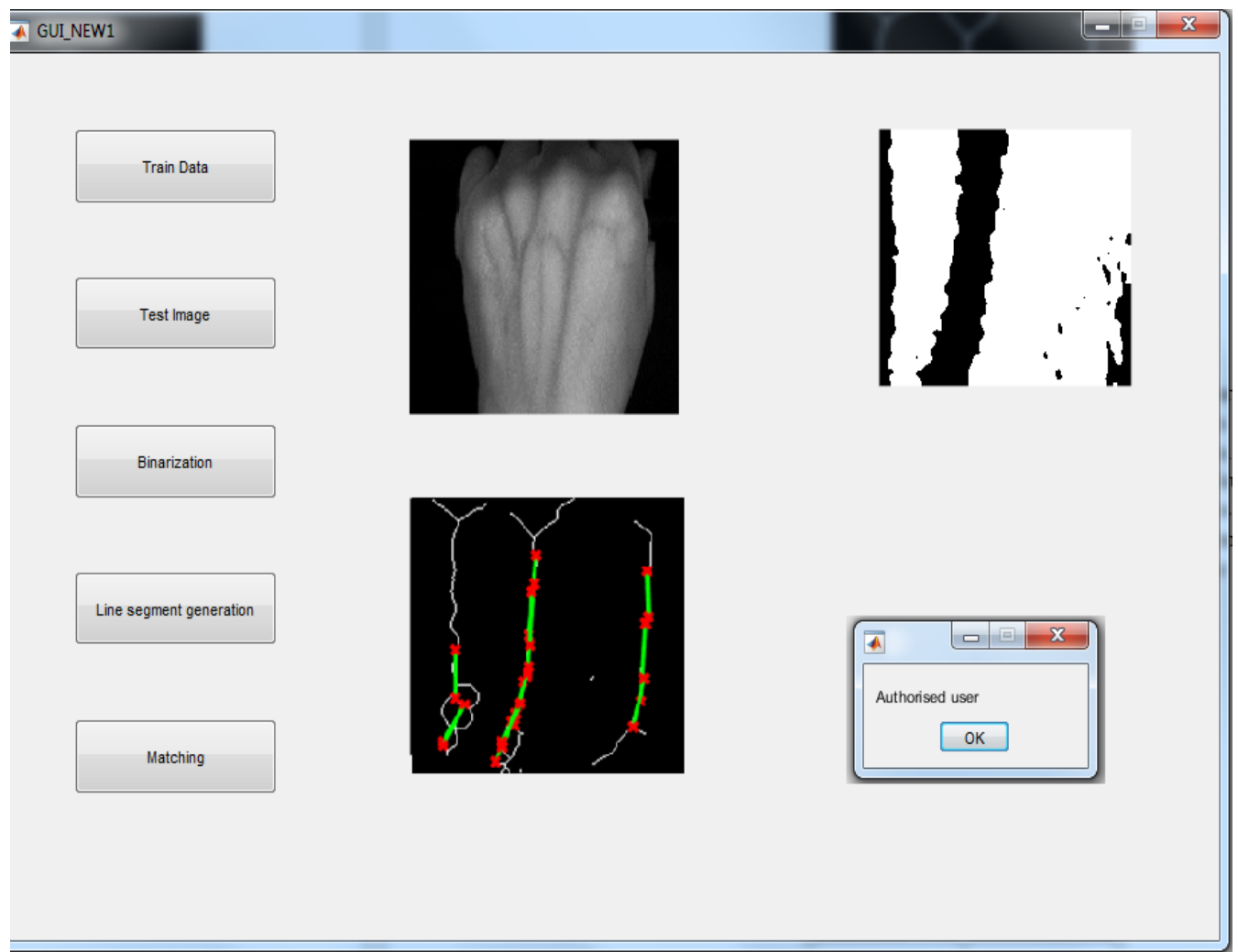

Fig 8. GUI

\section{CONCLUSION}

The Dorsal Hand Vein system thus proposed has an advantage that the region of interest is selected around the centroid which minimizes the translational effects making it more stable. Future work involves working on a larger database and considering the factors affected by age and various diseases. Minimization of the rotational effects can also be considered for improving the performance of the system.

\section{REFERENCES}

[1] P. Macgregor and R. Welford, "Veincheck: Imaging for Security and Personel Identification”, Advanced Imaging, Vol.6, pp. 52-56, January 1991.

[2] C. Colbert ,’Knuckle Profile Identity Verification system”, US Patent no.5862246 , Jan-1999.

[3] Chih -Lung Lin and Kuo-Chin Fan, "Biometric Verification Using Thermal Images of Palm-Dorsa Vein Patterns”,IEEE,Vol.14, pp.199-213,Feb-2004.

[4] Li Xueyan and Guo Shuxu , "The Fourth Biometric - Vein Recognition", Pattern Recognition Techniques, Technology and Applications, Peng-Yeng Yin (Ed.), ISBN:978-953-7619-24-4, In Tech., 2008

[5] Ajay Kumar, K. Venkata Prathyusha, "Personal Authentication using Hand Vein Triangulation and Knuckle Shape", IEEE Transactions on Image Processing , vol. 38, pp. 2127-2136, September 2009.

[6] M.Heenaye-Mamode Khan, R.K. Subramanian, and N. A. Mamode Khan, "Low Dimensional Representation of Dorsal Hand Vein Features Using Principle Component Analysis (PCA)" , International Journal of Computer, Electrical, Automation, Control and Information Engineering Vol:3, Issue No. 1, pg. No. 198-204, 2009.

[7] M. Rajalakshmi, R. Rengaraj , "Biometric authentication using near infrared images of palm dorsal vein patterns ." International Journal of Advanced EngineeringTechnology , E-ISSN 0976-3945 , IJAET/Vol.II/ Issue IV/ 384-389, October-December, 2011.

[8] Chih-Bin Hsu; Shu-Sheng Hao ; Jen-Chun Lee, "Personal authentication through dorsal hand vein patterns”, Opt. Eng. 50(8), 087201 doi:10.1117/1.3607413, July 28, 2011.

[9] C. Nandini ,Ashwini C, Medha Aparna, Nivedita Ramani, Pragnya Kini, Sheeba k , "Biometric Authentication by Dorsal Hand Vein Pattern ",International Journal of Engineering and Technology, ISSN: 2049-3444, Volume 2, Issue No. 5, , May, 2012.

[10] G. Sathish Dr. S.V. Saravanan Dr. S. Narmadha Dr. S. Uma Maheswari, " Personal Authentication System using Hand Vein Biometric "IJCTA International Journal of Computer Technology \& Applications, ISSN:2229-6093, Vol. no. 3, Pg no. 383-391, Jan.-Feb. 2012.

[11] V Evelyn Brindha, " Biometric Template Security using Dorsal Hand Vein Fuzzy Vault" , J Biomet Biostat (JBMBS), ISSN:21556180 , Volume 3 , Issue 4, doi:10.4172/2155-6180.1000145, 2012. 
[12] V. H. Yadav, "Dorsal hand vein Biometry by Independent component analysis." International Journal on computer Science and Engineering (IJCSE). ISSN : 0975- 3397, Vol.4 ,Issue No.07, Pg No: 1338-1344, July 2012.

[13] Ricardo Janes, Augusto Ferreira Brand, “A Low Cost System for Dorsal Hand Vein Patterns Recognition Using Curvelets” 2014 First International Conference on Systems Informatics, Modelling and Simulation, 2014.17 978-0-7695-5198-2, IEEE DOI 10.1109/SIMS.2014.17, 2014

[14] A. Djerouni, H. Hamada,A.Loukil, and N. Berrached , “ Dorsal Hand Vein Image Contrast Enhancement Techniques “ IJCSI International Journal of Computer Science Issues, ISSN (Print): 1694-0814 | ISSN (Online): 1694-0784, Vol. 11, Issue 1, No 1, , January 2014.

[15] Dipti Verma , Dr.Sipi Dubey , " A survey on Biometric authentication techniques using Palm Vein feature" , Journal of Global Research in Computer Science, Volume 5, No. 8, pg no. 5-8, August 2014.

[16] K. Premalatha*, T. Anantha Kumar, A.M. Natarajan, " A Dorsal Hand Vein Recognition-based on Local Gabor Phase Quantization with Whitening Transformation" , Defence Science Journal, Vol. 64, Issue No. 2, pp. 159-167, March 2014.

[17] Abiramasundari , S.Sasidevi , " Hand Dorsal Vein Recognition based on discrete Wavelet transforms ", International Journal of Engineering Research-Online, ISSN: 2321-7758, Vol.3., Issue.3, pg. no. 72-77, 2015.

[18] Ruchika Solanki, Vineet Khanna, " Vein Biometrics Identification Techniques and Challenges ", International Journal of Latest Trends in Engineering and Technology (IJLTET), Vol. 5 , Issue 4, pg. No. 448-456, July 2015.

\section{AUTHORS PROFILE}

Author 1 Name: Humairah Hamid, Student M.Tech - ECE, Amity University Haryana

Author 2 Name: V.K.Narang, Professor \& Director, Amity University Haryana 